\title{
BIBECHANA
}

ISSN 2091-0762 (Print), 2382-5340 (Online)

Journal homepage: http://nepjol.info/index.php/BIBECHANA

Publisher: Department of Physics, Mahendra Morang A.M. Campus, TU, Biratnagar, Nepal

\section{Diffusion of oxytocin in water: a molecular dynamics study}

\author{
Khimananda Acharya, Rajendra Prasad Koirala, Nurapati Pantha* \\ Central Department of Physics, Tribhuvan University, Kathmandu, Nepal \\ *Email: mrnurapati@gmail.com
}

\section{Article Information:}

Received: June 6, 2020

Accepted: July 11, 2020

Keywords:

Diffusion

Molecular Dynamics

Mean Square Displacement

Activation energy

\begin{abstract}
Classical molecular dynamics simulation is performed to estimate the diffusion coefficient of oxytocin in water at different temperatures, $288 \mathrm{~K}$, $300 \mathrm{~K}, 313 \mathrm{~K}, 323 \mathrm{~K}$, using GROningen Machine for Chemical Simulations (GROMOCS). The simulation is carried out using GROMOS43A1 force field and extended simple point charge (SPC/E) water model. The stability of the system is evaluated from energy profile of potential and kinetic energy, which assures well equilibrated molecular system. The selfdiffusion coefficient of oxytocin and water is obtained from Einstein's relation and binary diffusion coefficient is obtained from Darken's relation. As temperature increases the diffusion coefficient also increases as per expectation. The diffusion coefficients of water from the present calculations agree well with the previously reported values, within the $10 \%$ of deviation. Furthermore, the activation energy has been studied using Arrhenius Plot.
\end{abstract}

DOI: https://doi.org/10.3126/bibechana.v18i1.29316

This work is licensed under the Creative Commons CC BY-NC License. https://creativecommons.org/licenses/by-nc/4.0/

\section{Introduction}

Biomolecules are the building blocks of living organisms. They have the specific physio-chemical configurations [1]. The structural variability and chemical conformation of the molecules determine the biological functioning in living cells. Biomolecules work independently as well as cooperatively to perform in various body mechanisms [2]. Proteins, nucleic acids, lipids and carbohydrates are the basic category of biomolecules, also called the macromolecules. Some other molecules are also characterized into this class; primary metabolites, secondary metabolites, natural and artificially synthesized products $[3,4]$.

The poly-condensation of amino acids residues forms a protein molecule. The carboxyl (-COOH) tail of an amino acid when covalently bonded to amine (NH2-) head of another amino acid by water elimination, a dipeptide molecule is formed $[5,6]$. 
Then, the condensation of other amino acids one after another in new carboxyl tail, a long polypeptide chain is formed, which will finally be a functional protein molecule. A long polypeptide chain cannot stay in linear form, i.e., primary structure, because of difficulty in structural stability [7]. To minimize the overall energy profile, the molecule folds into various shapes, basically in alpha-helices and beta-turns. Hydrogen bonds, salt bridges; and hydrophobic and hydrophilic interaction takes place to provide different stable structures of protein molecules [4].

Oxytocin is a short peptide chain of nine amino acids (a nanopeptide) with the sequence cysteinetyrosine-isoleucine-glutamine-asparagine-cysteineproline-leucine-glycine (Cys Tyr Ile Gln Asn Cys Pro Leu Gly, or CYIQNCPLG) with additional amide (-NH2) in the carboxyl terminus of glysine residue [8] as shown in Fig 1(i) and its space-filling calotte $(\mathrm{CPK})$ model for the visualization is shown in Fig. 1(ii). Its molecular formula is $\mathrm{C}_{43} \mathrm{H}_{66} \mathrm{~N}_{12} \mathrm{O}_{12} \mathrm{~S}_{2}$ with its molecular weight $1007 \mathrm{~g}$ per 193 moles (i.e., 1007:193 g/mole). It has white powdery color and is soluble in water and in butanol. A disulphide bridge is present first and sixth cysteine residues of the molecule $[9,10]$.

Oxytocin is also known as love hormones responsible for the feeling love and satisfaction. It plays role in social bonding, sexual reproduction in both sexes, milk production etc. It is best known for its role in childbirth and breast feeding [12]. During labor large amount of oxytocin release causing contraction of the uterus to facilitate birth. When the contractions are not strong to compress the blood vessels, postpartum hemorrhage (PPH) can menace women's life. In this condition uterotoxic medicine like oxytocin is given to stimulate contraction and stop the bleeding $[11,13]$.

Oxytocin hormone is synthesized in pituitary gland. It is, then, released to the blood stream through the posterior lobe of the gland. Some neurons and other parts of brain also produce oxytocin $[14,15]$. Liquid contents of living body, like water, contribute to transport these hormones to various parts of the cell [16]. We believe that the study of transport properties of this hormone in water is very important to understand its mechanical properties in aqueous medium. Furthermore, diffusion coefficient of nitric oxide in water had been observed through the same technique [17]. To our best knowledge, the transport properties of oxytocin in water have not been studies yet. We have performed the molecular dynamics to deal the transport properties of oxytocin.

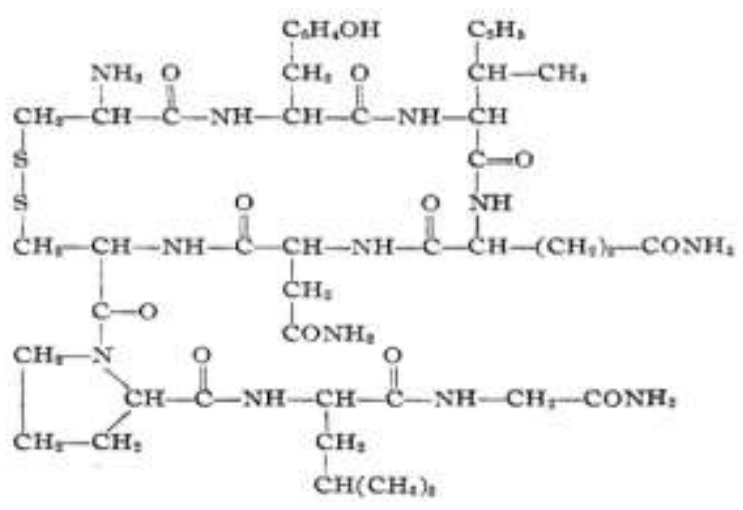

(i)

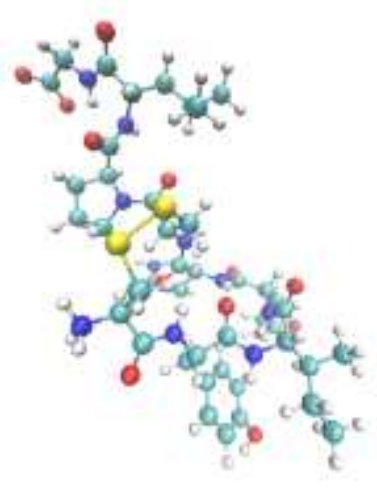

(ii)

Fig.1: (i) Molecular structure of an oxytocin molecule [11] (ii) CPK model of the oxytocin molecule.

\section{Diffusion}

Diffusion is the random process in which the matter is transported from one part of a system to another. 
Matter is transported always from higher concentration to the lower concentration. This occurs often due to the concentration inhomogeneity and thermal agitation of the particles in a medium. Diffusion is an imperative process within the human body and it is indispensable to transport of molecule within organ like lungs, kidneys, stomach, eyes etc. We realize several phenomena regarding the diffusion; like mixing of smoke in air, sugar in water, dust in air etc $[18,19]$. In this work, we estimate the selfdiffusion and binary diffusion coefficient of oxytocin in water.

The diffusion in a homogeneous system without any chemical concentration gradient exists in it is referred the self-diffusion and the corresponding diffusion coefficient is known as self-diffusion coefficient. The extent of diffusion is physically measured in terms of self-diffusion coefficient. It is calculated using Einstein's relation [20,21],

$D=\lim _{t \rightarrow \infty} \frac{\left\langle[r(t)-r(0)]^{2}\right\rangle}{6 t}$

The term $r(t)-r(0)$ in equation (1) is the displacement of particle from the reference position at time $t,[r(t)-r(0)]^{2}$ is the square of displacement, and $\langle\ldots\rangle$ represents the ensemble average, thus, the expression $\left\langle[\mathrm{r}(\mathrm{t})-\mathrm{r}(0)]^{2}>\right.$ is the mean square displacement (MSD) of the particle.

For the system with two different substances, binary diffusion takes place. It is measured in terms of diffusion coefficient called binary or mutual diffusion coefficient [22].

$D_{A B}=N_{B} D_{A}+N_{A} D_{B}$

where, $D_{A B}$ is Binary diffusion coefficient, $D_{A}, D_{B}$ $=$ Self diffusion coefficient of species $\mathrm{A}$ and $\mathrm{B}$ respectively, and $N_{A}, N_{B}=$ mole fraction of species $\mathrm{A}$ and $\mathrm{B}$ respectively.

The diffusion phenomenon is the temperature dependence phenomena. The mathematical relation for the temperature dependence is given by Arrhenius equation [23].

$D=D_{0} e^{\frac{-E_{a}}{R T}}$
Where, $\mathrm{D}$ is diffusion coefficient, $\mathrm{E}_{\mathrm{a}}$ is activation energy, $\mathrm{D}_{0}$ is pre-exponential factor, $\mathrm{R}$ is universal gas constant and $\mathrm{T}$ is absolute temperature.

\section{Computational Details}

\section{System setup}

A PDBID 2MGO.pdb was taken from the protein data bank (pdb), an authorized pdb website (www.rcsb.org). The molecule was in complete form as our requirement, so no modification was done in the original structure. The topology and parameters for the system setup and molecular dynamics (MD) simulations were taken from GROMOS43A1 force field [23]. While considering the water model, we should care on its compatibility with the force field parameter we have used [25]. The molecule was solvated into a cubic box of $4.07 \times 4.07 \times 4.07 \mathrm{~nm}^{3}$ dimension with SPC/E [26] water model by GROMACS 5.1.1 [27] command. The molecule was naturally chargeless, so extra ions have not been added. There were 2167 water molecules and one oxytocin molecule in the simulation box as shown in Fig. 2 .

Fig. 2: Oxytocin molecule solvated in a PBC box.

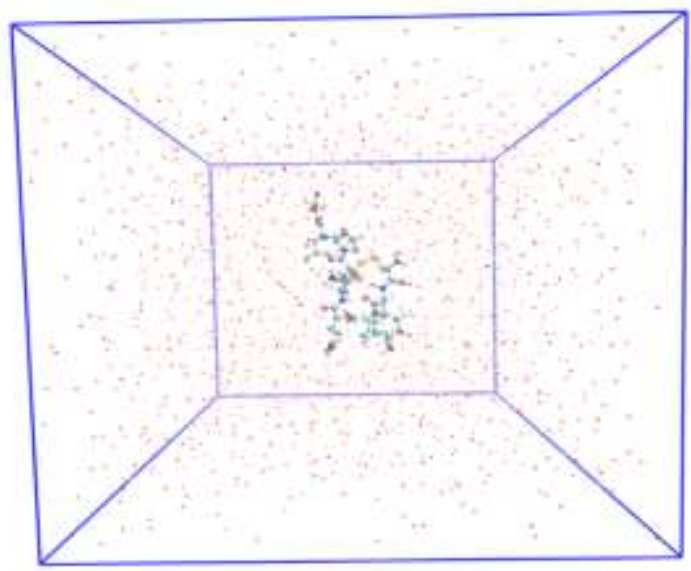

\section{Molecular dynamics simulations}

All atom molecular dynamics simulation was propagated to study the various mechanical properties of Oxytocin molecule in water. 
GROMOS43A1 force field was used for the entire simulation. Three basic steps: energy minimization, system equilibration and production run were carried out to deal the targeted properties.

The original pdb structure has been estimated from $\mathrm{x}$-ray diffraction (XRD) or nuclear magnetic resonance (NMR) techniques. The assigned coordinates for the atoms in the molecule might have steric hindrance due to the mechanical errors. Moreover, hydrogen atoms are absent in original pdb, and they are added by the molecular system building software. Therefore, before begin our MD run, we must be sure that there is no any inappropriate geometry otherwise force acting on particle will be very large and system will be out of equilibrium and MD fails. In order to get solved this inappropriate geometry and kinetic energy; system must be subjected to the process of energy minimization. In our system because of fast and reliable performance, we have decided to choose steepest-descent algorithm [29].

Energy minimization run is often performed in absolute zero Kelvin temperature, which is practically unachievable and unreliable for the natural environment. So, the molecular environment is so created that can be resembled in the practice in room temperature or for any natural process. We basically maintain the system that follows the statistical ensembles with appropriate macroscopic parameters; pressure, temperature and volume [30,31]. Pressure coupling was made to set pressure 1 bar using barendsen barostat. For box re-scaling isothermal compressibility is set to 4.5 $\times 10^{-5} \mathrm{bar}^{-1}$. Particle Mesh Ewald (PME) for coulomb interaction with cut off distance $1.0 \mathrm{~nm}$ and Fourier spacing of 0.3 was used. V-rescaling has been used for temperature coupling. Equilibration run was performed at four different temperatures $288 \mathrm{~K}, 300 \mathrm{~K}, 313 \mathrm{~K}$, and $323 \mathrm{~K}$ and was run $20 \mathrm{~ns}$ for each system.

The appropriate system prepared from the equilibration run was propagated to study the molecular diffusion in water at assigned temperature above. Production run was performed in NVT ensemble. Each of the system was run 60 ns time period.

\section{Results and Discussion}

We present and discuss about the energy profiles, self-diffusion coefficients of both water and oxytocin; and the output of self-diffusion coefficient is used to evaluate binary diffusion coefficients. Finally, the activation energy of corresponding systems is estimated for each system.

\section{Energy minimization and system equilibration}

The fundamental requirement of MD simulation is to bring the molecular system in minimum potential energy so that the atoms in the molecule get free from atomic hindrance. The best way to achieve this condition is energy minimization run and analyzing the outcomes. Energy minimization run is always run at absolute zero temperature, which ensures the minimum potential energy. Fig. 3 shows the potential energy curve during the minimization run. The potential energy has been well converged and we confirmed the system to proceed for the post processing. We found potential energy $-1.2015872 \times 10^{4} \mathrm{~kJ} \mathrm{~mol}^{-1}$ in the most condition and steepest descent converged to $\mathrm{F}_{\max }<$ $50 \times 10^{4} \mathrm{~kJ} \mathrm{~mol}^{-1}$.

Before commencing the MD production run, the molecular system should be maintained at a thermodynamic ensemble. This can be done by MD equilibration run and the outcomes were evaluated determining some thermodynamic parameters like pressure, temperature etc. These parameters have been assigned to resemble the cellular environment in human body. The best way of finding the stability of the molecular system is the calculation of density, which was not assigned for the system. We have calculated density of water and entire system including the oxytocin after equilibration run. The variation of density of water and entire system at different temperatures has been presented in table 1. Because of infinite dilution, the mixture 
has similar order of density with that of pure water. The decreasing pattern of density on increasing temperature ensures that the system is appropriately equilibrated and is suitable for the further calculation.

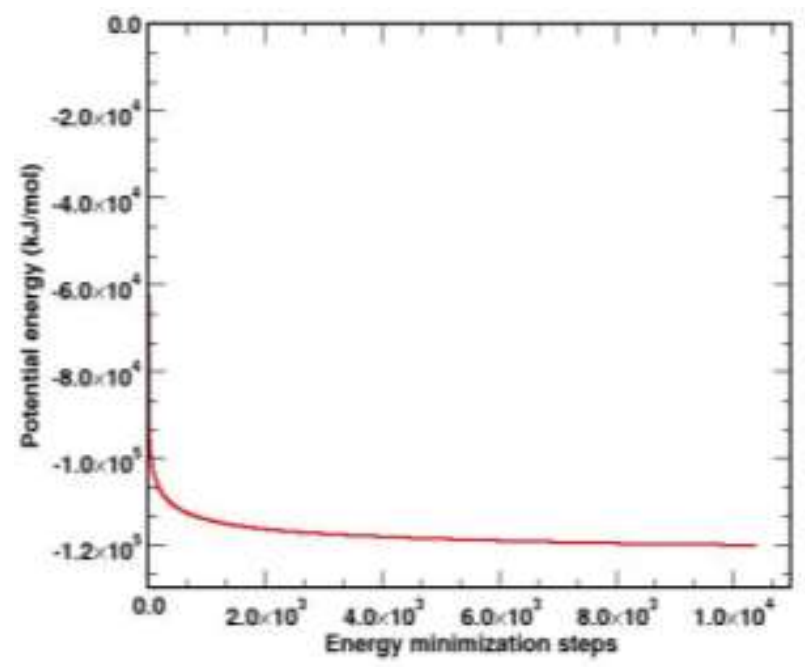

Fig. 3: Potential energy after energy minimization.

\section{Energy profile}

We have studied energy profile under the GROMOS43A1 force field in MD simulation. When the system is in MD simulations, several interactions have been taken to calculate energy. The energy profile is contributed by many bonded and non-bonded interactions. Bond interactions are bond stretching, bond angle vibration, proper dihedral and improper dihedral whereas nonbonded interactions are coulomb interaction and L$\mathrm{J}$ interaction. The energy profile is the graphical manifestation of these various energy interactions.

To reduce the computational cost, we have kept bond length constant in our simulation with the help of constraint algorithm LINCS. Therefore, there is no energy profile due to bond stretching. Also we found that bonded interaction energy was positive. The coulomb interaction and L-J interaction are the major contribution on the non-bonded interaction.
These interactions were calculated using the list of non-bonded atom within the certain region. We use periodic boundary condition for such a purpose. The SR coulomb interaction has negative value and has dominant contribution on total potential energy. The value of L-R coulomb and coulomb reciprocal energy are significantly small. Within the cutoff region, the L-J interaction has significant role, however, (L-R) L-J interaction has negligible contribution on total energy. The energy profiles of the system at four different temperatures have been studied. The energy profile for all the interaction energy with total energy at $300 \mathrm{~K}$ temperature is shown in Fig. 4.

\section{Self-diffusion coefficient of water and oxytocin}

Self-diffusion coefficients of water and oxytocin have been calculated at four different temperatures; $288 \mathrm{~K}, 300 \mathrm{~K}, 313 \mathrm{~K}, 323 \mathrm{~K}$ using the Einstein's relation in equation (1). The slope of corresponding mean square displacement (MSD) was utilized to determine the self-diffusion coefficients in both systems. Fig. 5(i) and 5(ii) show the mean square displacement curves for water and oxytocin respectively.

We have carried out $60 \mathrm{~ns}$ MD simulation for each temperature; however the linear relationship has to be required to draw the value of slope in MSD versus time graph. So, we have selected 2 ns simulation run in the beginning to get the better statistics. The MSDs, thus, obtained were found linearly increasing with respect to time, and in turn increased the self-diffusion coefficients. The selfdiffusion coefficient of water was compared with previously reported experimental value and was found good agreement within 10\%. The better agreement at higher temperature indicates that the force field parameters could have been calculated in these temperature regimes which are relevant to physiological phenomenon. 
Table 1: Temperatures and corresponding densities of system after equilibration run.

\begin{tabular}{|c|c|c|c|}
\hline $\begin{array}{c}\text { Coupling } \\
\text { temperature } \\
(\mathbf{K})\end{array}$ & $\begin{array}{c}\text { Equilibrium } \\
\text { temperature } \\
(\mathbf{K})\end{array}$ & $\begin{array}{c}\text { Equilibrium density } \\
\left(\mathbf{k g ~ m}^{-\mathbf{3}}\right)\end{array}$ & $\begin{array}{c}\text { Density of } \\
\text { water }\left(\mathbf{k g ~ m}^{\mathbf{3}} \mathbf{)}\right. \\
{[\mathbf{3 2}]}\end{array}$ \\
\hline 288 & $287.983 \pm 0.007$ & $1008.71 \pm 0.07$ & 999.13 \\
\hline 300 & $299.983 \pm 0.007$ & $1002.87 \pm 0.05$ & 996.56 \\
\hline 313 & $313.005 \pm 0.011$ & $995.46 \pm 0.02$ & 992.28 \\
\hline 323 & $323.995 \pm 0.011$ & $998.96 \pm 0.03$ & 988.11 \\
\hline
\end{tabular}

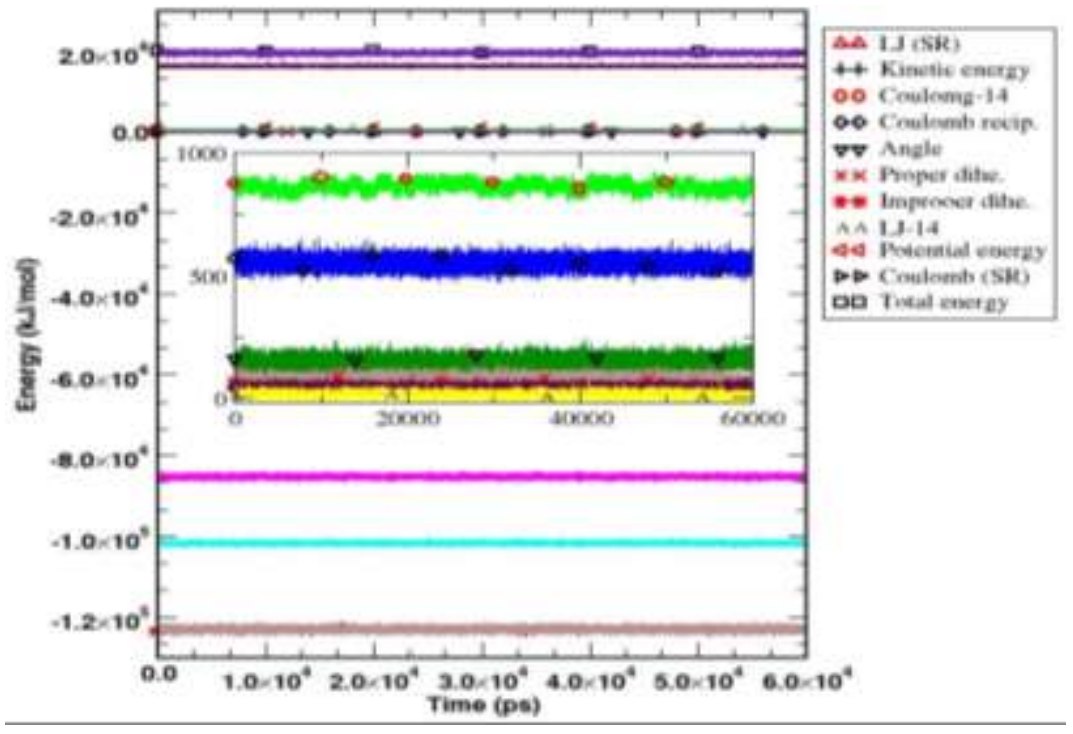

Fig. 4: Total energy profile of oxytocin at $300 \mathrm{~K}$.

The simulated values and experimental values of self-diffusion coefficient of water at different temperatures are listed in the table 2 . We also have calculated self-diffusion coefficient of oxytocin from the MSD graph of oxytocin molecule. The simulated values of self-diffusion coefficient of oxytocin at different temperatures are listed in the table 3. In both systems, the self-diffusion coefficients are obtained as, $\mathrm{D}_{323} \mathrm{~K}>\mathrm{D}_{313} \mathrm{~K}>\mathrm{D}_{300}$ $\mathrm{K}>\mathrm{D}_{288} \mathrm{~K}$.
While simulating macromolecule/s in a box, one needs to be careful about its size effect. It is a usual practice to consider the smallest possible simulating box and enlarge the space for moving molecules by applying periodic boundary condition (PBC). However, the size of the box should be sufficient to maintain the basic criteria of molecular simulations. The rule of thumb for this requirement says that the cut-off distance of different interactions under consideration should be less than the half the box size [24]. Furthermore, previous 

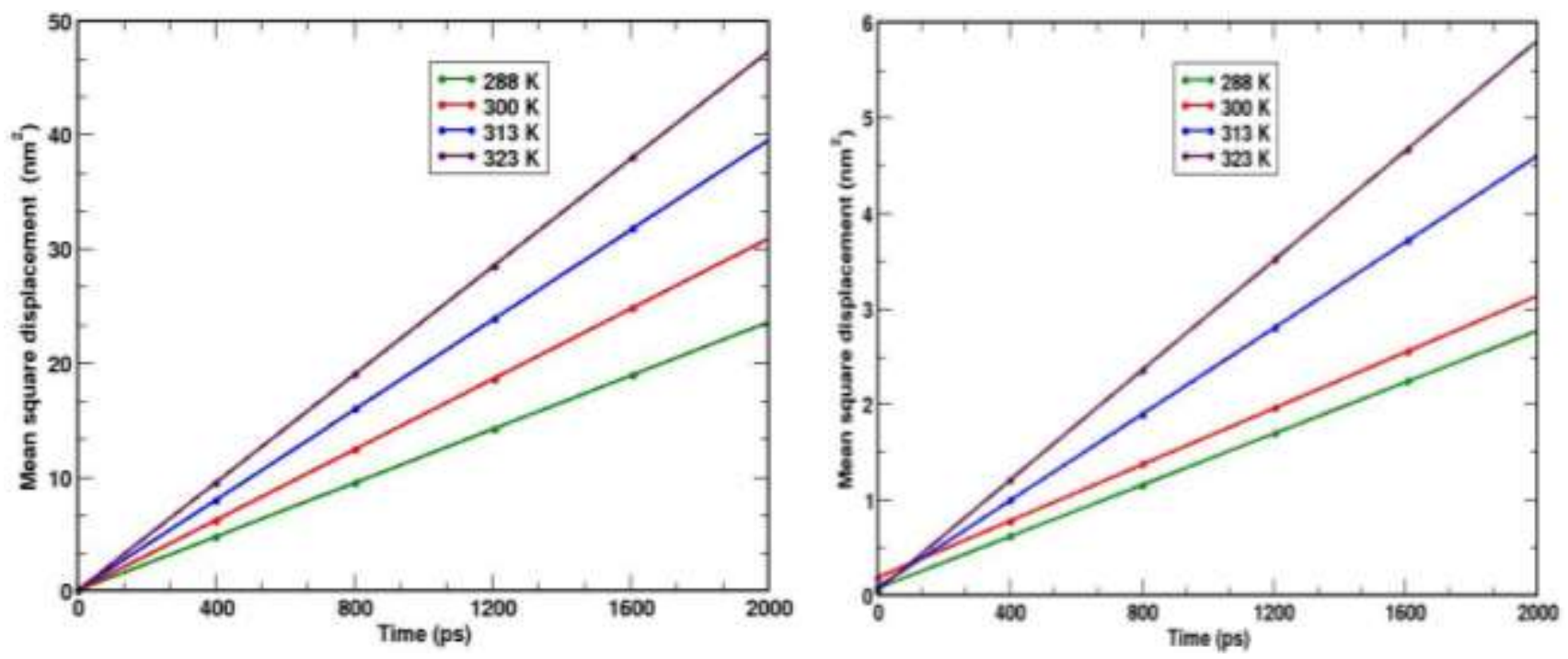

Fig. 5: MSD vs time plot of (i) water (ii) oxytocin at different temperatures.

Table 2: Simulated and experimental self-diffusion coefficients of water at four different temperatures.

\begin{tabular}{|c|c|c|c|}
\hline $\begin{array}{c}\text { Temperature } \\
\text { K }\end{array}$ & $\begin{array}{c}\text { Simulated diffusion } \\
\text { coefficient } \\
D_{w}^{\mathrm{s}}\left(\mathbf{1 0}^{-10} \mathrm{~m}^{2} / \mathrm{s}\right)\end{array}$ & $\begin{array}{c}\text { Experimental diffusion } \\
\text { coefficient } \\
D_{w}^{E}\left(1^{-10} \mathbf{m}^{2} / \mathrm{s}\right)[33]\end{array}$ & Difference \% \\
\hline 288 & $19.16 \pm 0.05$ & 17.77 & 9.39 \\
\hline 300 & $25.72 \pm 0.05$ & - & - \\
\hline 313 & $32.87 \pm 0.10$ & 32.40 & 1.43 \\
\hline 323 & $39.39 \pm 0.08$ & 39.68 & 0.74 \\
\hline
\end{tabular}

studies have shown that as far as these minimum criteria are maintained, the size of the box does not affect the main conclusions of the results [34]. We have taken care of size of the oxytocin molecule and also the cutoff distances, which are less than half-size of the simulating box in each dimension.

\section{Binary diffusion coefficient}

The self-diffusion coefficients obtained for water and oxytocin were used to find the binary diffusion coefficients of oxytocin in water by using Darken's relation in equation (2). The calculated values of binary diffusion coefficients were found increasing on increasing the temperature. When temperature increases there is increase in thermal energy which results the decrease in density of the system and hence there is increase in diffusion coefficient. The estimated binary diffusion coefficients of oxytocin in water have been presented in table 4 . 
Table 3: Simulated values of self-diffusion coefficients of oxytocin at four different temperatures.

\begin{tabular}{|c|c|}
\hline $\begin{array}{c}\text { Temperature } \\
\mathbf{K}\end{array}$ & $\begin{array}{c}\text { Self-diffusion Coefficient } \\
\mathbf{D}_{\mathbf{0}}^{\mathbf{s}} \mathbf{( \mathbf { 1 0 }}\end{array}$ \\
\hline 288 & $2.24 \pm 0.32$ \\
\hline 300 & $2.45 \pm 0.29$ \\
\hline 313 & $3.75 \pm 0.26$ \\
\hline 323 & $4.79 \pm 0.01$ \\
\hline
\end{tabular}

Table 4: Simulated binary diffusion coefficients at four temperatures.

\begin{tabular}{|c|c|}
\hline $\begin{array}{l}\text { Temperature } \\
\text { K }\end{array}$ & $\begin{array}{l}\text { Binary Diffusion Coefficient } \\
D_{0}{ }^{\mathrm{s}}\left(10^{-10} \mathrm{~m}^{2} / \mathrm{s}\right)\end{array}$ \\
\hline 288 & 2.23 \\
\hline 300 & 2.46 \\
\hline 313 & 3.76 \\
\hline 323 & 4.81 \\
\hline
\end{tabular}

\section{Temperature dependency of diffusion}

We obtain activation energy from the slope of diffusion versus reciprocal of time as suggested by the equation (3), $D=D_{0} e^{\frac{-E a}{N_{A} k_{B} T}}$, and $\mathrm{R}=\mathrm{N}_{\mathrm{A}} \mathrm{k}_{\mathrm{B}}$, where $\mathrm{N}_{\mathrm{A}}$ is Avogadro number whose value is $6.023 \times 10^{-23} \mathrm{~mol}^{-1}$ and $\mathrm{k}_{\mathrm{B}}$ is the Boltzmann constant carrying the value $1.38 \times 10^{-23} \mathrm{JK}^{-1}$, meaning of remaining parameters are explained in equation (3). On taking the natural logarithm for the expression, we get,

$\ln D=\ln D_{0}-\frac{E_{a}}{N_{A} k_{B} T}$

Then, the activation energy $E_{a}$ for diffusion was obtained from the slope of $\ln D$ versus $\frac{1}{T}$, called the Arrhenious plot as,

$E_{a}=-N_{A} k_{B} \frac{\partial \ln D}{\partial(1 / T)}$

The intercept when extrapolated to the $\frac{1}{T} \rightarrow 0$ in the Arrhenius plot gives the pre-exponential factor.

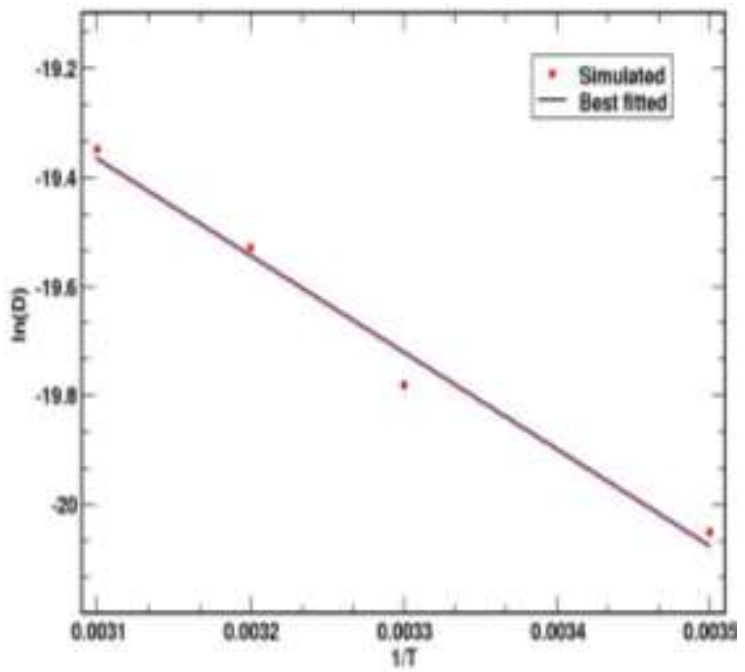

(i)

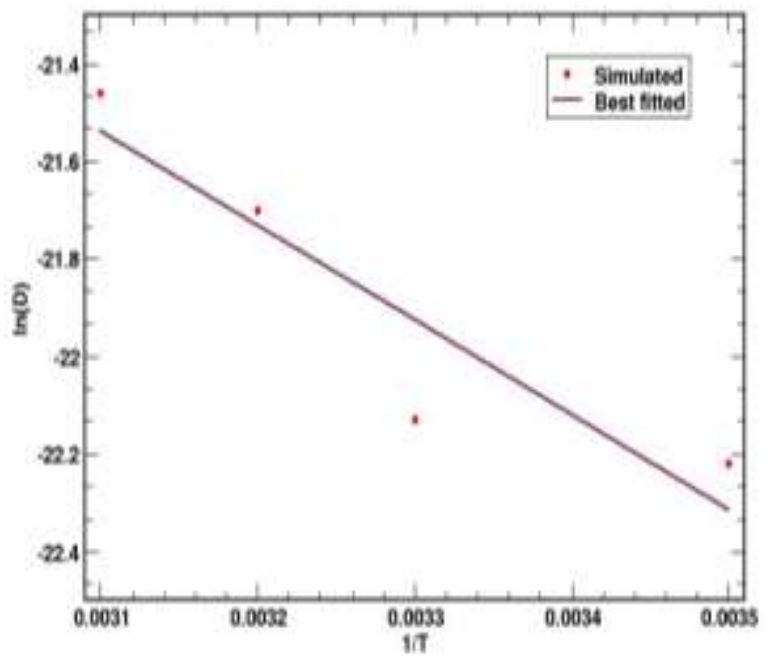

(ii)

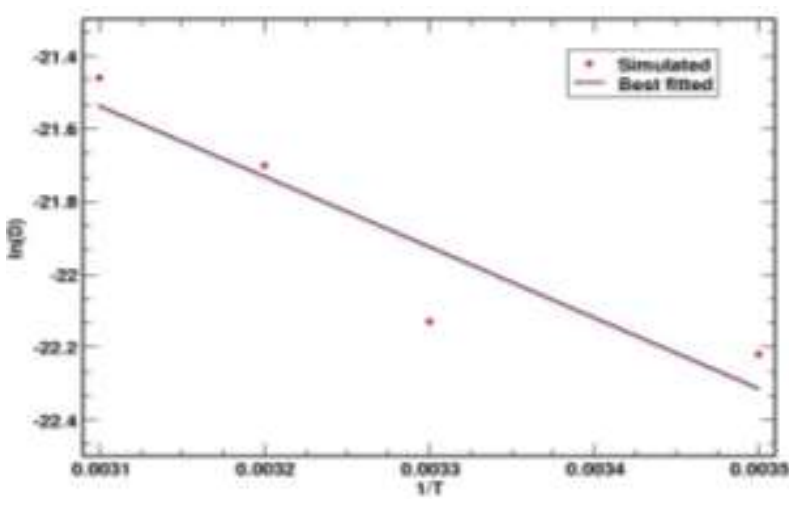

(iii)

Fig. 6: Arrhenius diagram for self-diffusion coefficient of (i) Oxytocin, (ii) Water and (iii) Binary mixture. 
The Arrhenius plot of simulated value of water is shown in Fig. 6(i), and that of oxytocin is 6(ii) and for their binary mixture is shown in Fig. 6(iii), which were used to determine the activation energy for each condition. From the table 5, it is clear that the activation energy for oxytocin molecule and binary mixture is found to be almost equal; this is due to the infinitesimal concentration of oxytocin in our system. Further, we found that there is an acceptable level of agreement between the simulated and experimental values of activation energy of water.

\section{Conclusions and concluding remarks}

The self-diffusion coefficient of water and oxytocin is obtained using Einstein's relation. The binary diffusion coefficient is obtained using Darken's relation. The results obtained for energy profile, the kinetic energy is proportional to absolute temperature, is as expected. The experimented and simulated value of self-diffusion coefficient of water for all temperature is within error $10 \%$. Diffusion coefficient of water for water and oxytocin are found increasing with increase in temperature. Furthermore, the dependency of diffusion with temperature is analyzed with Arrhenius plot. Activation energy is also calculated from the slope of these plots. We have found that the activation energy of water obtained from simulated data is differing from experimented value with an error of $17.18 \%$. We do not have any experimental result for diffusion of oxytocin at present time to compare our final result; however, we can be sure on our accuracy with comparing the results of simulated and experimental self-diffusion coefficient of water.

\section{Acknowledgements}

K. Acharya acknowledges the partial support from University Grants Commission (UGC), Nepal. R.P.K. acknowledges the partial financial support from the Nepal Academy of Science and Technology (NAST).

\section{References}

[1] P. Narayanan, Essentials of biophysics, New Age International Publishers, New Delhi (2010).

[2] C. M. Venkatachalam, G. N. Ramachandran, Conformation of polypeptide chains, Annual Review of Biochemistry 38(1) (1969) 45-82.

[3] C. Mura, C. E. McAnany, An introduction to biomolecular simulations and docking, Molecular Simulation 40 (10-11) (2014) 732-764. https://doi.org/10.1080/08927022.2014.935372

[4] W. H. Brown, T. Poon, Introduction to organic chemistry, John Wiley \& Sons (2014).

[5] R. Cotterill, Biophysics: an introduction, John Wiley \& Sons (2003).

[6] G. Ramachandran, V. Sasisekharan, Ado, Protein Chcm, 23 (1968) 283.

[7] S. B. Kent, Total chemical synthesis of proteins, Chemical Society Reviews 38(2) (2009) 338-351.

https://doi.org/10.1039/B700141J

[8] V. J. Hruby, M. S. Chow, D.D. Smith, Conformational and structural considerations in oxytocin-receptor binding and biological activity, Annual Review of Pharmacology and Toxicology 30(1) (1990) 501-534.

[9] C. A. Burtis, E. R. Ashwood, D. E. Bruns, Tietz textbook of clinical chemistry and molecular diagnostics-e-book, Elsevier Health Sciences (2012).

[10] https://pubchem.ncbi.nlm.nih.gov/compound/Oxytocin

[11] V. D. Vigneaud, C. Ressler, C. J. M. Swan, C. W. Roberts, P. G. Katsoyannis, S. Gordon, The synthesis of an octapeptide amide with the hormonal activity of oxytocin, Journal of the American Chemical Society 75(19) (1953) 48794880. https://doi.org/10.1021/ja01115a553

[12] S. C. Herpertz, K. Bertsch, Oxytocin effects on brain functioning in humans, Biological Psychiatry 79(8) (2016) 631-632. https://doi.org/10.1016/J.blopsych.2016.02.004

[13] F. T. Buisman-Pijlman, N. M. Sumracki, J. J. Gordon, P. R. Hull, C. S. Carter, M. Tops, Individual differences underlying susceptibility to addiction: role for the endogenous oxytocin system, Pharmacology Biochemistry and Behavior 119 (2014) 22-38. https://doi.org/10.1016/j.pbb.2013.09.005

[14] E. Sausville, D. Carney, J. Battey, The human vasopressin gene is linked to the oxytocin gene and 
is selectively expressed in a cultured lung cancer cell line, Journal of Biological Chemistry 260 (18) (1985) 10236-10241.

[15] H. E. Ross, C. D. Cole, Y. Smith, I. D. Neumann, R. Landgraf, A. Z. Murphy, L. J. Young, Characterization of the oxytocin system regulating affiliative behavior in female prairie voles, Neuroscience 162(4) (2009) 892-903. https://doi.org/10.1016/j.neuroscience.2009.05.055

[16] R. P. Koirala, H.P. Bhusal, S. P. Khanal, N. P. Adhikari, Effect of temperature on transport properties of cysteine in water, AIP Advances 10(2) (2020) 025122. https://doi.org/10.1063/1.5132777

[17] S. Pokharel, N. Pantha, and N. Adhikari, Diffusion coefficients of nitric oxide in water: A molecular dynamics study, International Journal of Modern Physics B 30 (27) (2016) 1650205. https://doi.org/10.1142/S0217979216502052

[18] J. Crank, The mathematics of diffusion 2nd Edition. Oxford Science Publications (1975).

[19] H. Hirakawa, Y. Kamei, M. Sugisaki, Y. Oishi, Relationship between Self-Diffusion and Interdiffusion in Gaseous Systems. Bulletin of the Chemical Society of Japan 46(9) (1973) 26592662. https://doi.org/10.1246/bcsj.46.2659

[20] D. Frenkel, B. Smit, Understanding molecular simulation: From algorithms to applications. Computational Sciences Series 1(2002) 1-638.

[21] J. Lekner, Summation of Coulomb fields in computer-simulated disordered systems, Physica A: Statistical Mechanics and its Applications 176(3) (1991) 485-498. https://doi.org/10.1016/0378-4371(91)90226-3

[22] L. S. Darken, Diffusion, mobility and their interrelation through free energy in binary metallic systems, Trans. Aime 175 (1948) 184-201.

[23] H. Mehrer, Diffusion in solids: fundamentals, methods, materials, diffusion-controlled processes, Springer Science \& Business Media (2007).

[24] M. Allen, D. Tildesley, Computer simulation of liquids, New York (1987).

[25] U. Dahal, N. P. Adhikari, "Molecular dynamics study of diffusion of heavy water in normal water at different temperatures," Journal of Molecular Liquids 167(2012) 34-39. https://doi.org/10.1016/j.molliq.2011.12.008

[26] H. J. C. Berendsen, J. R. Grigera, T. P. Straatsma, The missing term in effective pair potential, Journal of Physical Chemistry 91 (1987) 62696271. https://doi.org/10.1021/j100308a038

[27] D. Spoel et al., GROMACS User Manual, version 5.1.1, 2016.

[28] H. J. Berendsen, J. V. Postma, W. F. van Gunsteren, A. R. H. J. DiNola, J. R. Haak, Molecular dynamics with coupling to an external bath, The Journal of Chemical Physics 81(8) (1984) 3684-3690. https://doi.org/10.1063/1.448118

[29] Gromacs user manual, http://manual.gromacs.org/documentation/2016.4/ manual-2016.4.pdf.

[30] J. N. Evans, Biomolecular NMR spectroscopy (No. 543.42 EVA), Oxford University (1995).

[31] E. B. Walton, K. J. VanVliet, Equilibration of experimentally determined protein structures for molecular dynamics simulation, Physical Review E 74(6) (2006) 061901. https://doi.org/10.1103/PhysRevE.74.061901

[32] M. Tanaka, G. Girard, R. Davis, A. Peuto, N. Bignell, Recommended table for the density of water between $0 \mathrm{C}$ and $40 \mathrm{C}$ based on recent experimental reports, Metrologia 38 (4) (2001) 301.

https://doi.org/10.1088/0026-1394/38/4/3

[33] M. Holz, S. R. Heil, and A. Sacco, Temperaturedependent self-diffusion coefficients of water and six selected molecular liquids for calibration in accurate $1 \mathrm{~h} \mathrm{nmr}$ pfg measurements, Physical Chemistry Chemical Physics 2 (2000) 4740- 4742. https://doi.org/10.1039/B005319H

[34] P. Mark and L. Nilsson, Structure and Dynamics of the TIP3P, SPC, and SPC/E Water Models at $298 \mathrm{~K}$, Journal of Physical Chemistry A 105 (2001) 9954-9960. https://pubs.acs.org/doi/10.1021/jp003020w 